This article is licensed under the Creative Commons Attribution-NonCommsticentives 4.0 Intera

\title{
A Single Nucleotide Polymorphism (rs1056629) in 3'-UTR of MMP-9 is Responsible for a Decreased Risk of Metastatic Osteosarcoma by Compromising its Interaction with microRNA-491-5p
}

\author{
Xiaodong Tian ${ }^{\mathrm{a}, \mathrm{b}} \quad$ Xuesong Zhang ${ }^{\mathrm{c}}$
}

aGraduate School, China Medical University, Shenyang, ${ }^{b}$ Department of Orthopedics, Central Hospital of Beipiao, Beipiao, 'Department of Orthopedics, The PLA General Hospital, Beijing, China

\section{Key Words}

rs1056629 • MMP-9 • Metastasis • Osteosarcoma • microRNA-491-5p

\begin{abstract}
Background: This study aimed to explore the roles of microRNA-491-5p (miR-491) and its target MMP9 in the control of metastasis of osteosarcoma (OS) as well as how the rs 1056629 polymorphism in the $3^{\prime}$ untranslated region (3'UTR) of MMP9 compromises the interaction between miR-491 and MMP9. Methods: We used bioinformatics tools to identify possible binding sites of miR-491 in the 3'UTR of MMP9 and 30 OS tissue samples were collected and divided into two groups based on the rs1056629 genotype (AA, N=20; $A C, N=8$; and CC $\mathrm{N}=2$ ). The expression of miR-491 and MMP9 were determined in those samples. Results: We found that the rs 1056629 polymorphism potentially compromised the interaction between miRNA and mRNA, which was subsequently confirmed by using the luciferase reporter system. we found that the expression of miR-491 was comparable among the genotype groups, whereas the expression of MMP9 was much higher in the AC/CC groups than in the AA group. Furthermore, 10 OS cell lines (OSA, MG-63, Saos-2, U2OS, SARG, KPD, OHS, HAL, ZK-58, and MHM) were genotyped for the rs1056629 polymorphism, and MG-63 (AA) and OSA (AC) were identified for the scratch test and Transwell assay that followed. In the MG-63 cells, transfection of the miR-491 mimics and MMP9 siRNA similarly and substantially down regulated the expression of MMP9, and miRNA and siRNA clearly suppressed the migratory and invasive ability. In the OSA cells, only MMP9 siRNA notably reduced the expression of MMP9 and decreased the migratory and invasive ability. The introduction of miR-491 mimics left the expression of MMP9 and the migratory and invasive ability intact. Conclusion: We found that the rs 1056629 polymorphism interfered with the interaction between MMP9 mRNA and miR-491 and is associated with the metastasis of OS cells.
\end{abstract}




\section{Introduction}

Generally found in youths between 10 and 25 years old, osteosarcoma (OS) is an aggressive bone malignancy originating from the mesenchyme cells. OS comprises $2.4 \%$ of pediatric malignancies and ranks eighth among the most common cancers in children [1]. Originating from primitive transformed mesenchymal cells, OS is an aggressive malignant neoplasm that produces malignant osteoids and exhibits the characteristics of osteoblastic differentiation [1]. Any bone of the human body could be affected by this neoplasia, and the general survival rate at five years is approximately 65 to $75 \%$. The main causes of death are pulmonary metastases, which are diagnosed by computed tomography (CT) in 35 to $45 \%$ of OS patients [2].

MicroRNAs (miRNAs) comprise a class of small non-coding RNAs consisting of 21 to 25 nucleotides that regulate target gene expression post transcriptionally [3]. MiRNAs have been reported to be functionally involved in the control of various biological processes including the proliferation, differentiation, invasion and apoptosis of cells [4]. MiRNAs have been shown to be crucial in cancer progression, and they provide novel targets for anticancer therapies [4]; they act as oncogenes to tumor suppressors, depending on the function of their target gene (s) [3,5-8]. Additional polymorphisms have been identified in microRNAs or its binding sites in mRNA, and they are becoming increasingly important in personalized medicine [9]. The polymorphisms in the 3'UTR might be found at or near the binding site of functional genes. By disturbing the microRNA - mRNA interaction, polymorphisms in the 3'UTR could influence gene expression. Compared with other SNPs in the 3'UTR, Yu et al. discovered that polymorphisms within predicted miRNA seed-complementarity sites in the 3'UTR possessed a functional significance, which might result from disruption of the interaction between miRNA and mRNA, and thereby release physiologically inhibited target genes [9].

Matrix metalloproteinase 9 is $92-k D a$ type IV collagenase (gelatinase B, MMP9), and it has fibronectin-like domains, which could bind collagen; it is able to degrade laminin and types I, V, IV, XI and VII collagen [10,11]. Growing evidence has indicated that malignant tumors have higher expression levels of MMP9 than non-invasive or benign tumors, and MMP9 is hypothesized to play a functional role in regulating tumor invasion or migration in vivo and in vitro $[12,13]$. An immunohistochemical analysis identified MMP9 to be strongly positive in metastastic lesions from children with osteosarcoma [14]. A single nucleotide polymorphism (SNP) (rs1056629) is located in the miR-491 binding site in the 3'UTR of MMP9, and it confers an increased risk for an atherosclerotic cerebral infarction [15]. We hypothesize that rs1056629 might interfere with the interaction between MMP9 mRNA and miR-491 and that the minor allele (C) might be associated with OS metastasis.

\section{Materials and Methods}

\section{Patient samples}

We recruited 30 OS patients, who received surgical intervention for the treatment of oS in the First Affiliated Hospital of China Medical University, and obtained biopsy specimens of human OS from primary lesions. Patients who received chemotherapy or radiotherapy were excluded from the study, and the specimens were used for diagnoses as well. The clinicopathological data of the recruited patients are summarized in Table 1. The study protocol was approved by the Institutional Review Board of China Medical University. Written informed consent was obtained from the patients recruited in the study.

\section{Genotyping}

The genotyping of the rs105662 polymorphism was performed using direct Sanger sequencing. The segment in the 3'UTR MMP9 containing the polymorphism was PCR amplified with the following primer set: forward 5' ATCCAAGTTTATTAGAAACACTCCA-3' and forward 5'-GGATGTCATAGGTCACGTAGCCCAC-3'. The purity of the PCR product was tested by agarose electrophoresis before sending it to the sequencing core on the campus of China Medical University. 


\section{Cellular Physiology Cell Physiol Biochem 2016;38:1415-1424 \\ \begin{tabular}{l|l|l|l|}
\hline DOI: 10.1159/000443084 & (c) 2016 The Author(s). Published by S. Karger AG, Basel \\
\hline
\end{tabular} \\ Published online: March 30, 2016 www.karger.com/cpb}

Tian/Zhang: rs1056629 Polymorphism Compromises Interaction Between MMP9 3'UTR and miR-491

\section{Real-Time PCR}

Total RNA was isolated from the os tissue samples or the cultured cells by using Trizol reagent (Invitrogen, Carlsbad, CA). Real-time PCR (QPCR) was performed in an Applied Biosystems 7500 RealTime PCR system (Applied Biosystems, Foster City, CA) following the manufacturers' protocol. We mixed a final $20 \mathrm{uL}$ volume of reagents consisting of $1 \mu \mathrm{L}$ of cDNA template, $1 \mu \mathrm{L}$ of each forward and reverse primer, $7 \mu \mathrm{L}$ of $\mathrm{H} 2 \mathrm{O}$ and $10 \mu \mathrm{L}$ of SYBR advantage qPCR Premix (Clontech, MountainView, CA, USA). The standard curve method was used to quantify the levels of miR-491 and MMP9 mRNA. We constructed the standard curves with a serially diluted standard template. To account for the amount of template in the reaction mixtures and the differences in reverse transcription efficiencies, we normalized to data to U6 and NADPH. The $2^{-\triangle \Delta \mathrm{Ct}}$ method was used to describe the expression of miR-491 or MMP9 mRNA normalized to the internal control.

\section{Cell culture and transfection}

In total, 10 OS cell lines (OSA, MG-63, Saos2, U20S, SARG, KPD, OHS, HAL, ZK-58, and MHM) were purchased from ATCC (Manassas, VA, USA) and genotyped for the rs105662 polymorphism, and OSA (A/C) and MG-63 (A/A) cells were used for the following functional analysis: the miR-491 mimics (5'- AGU GGG GAA CCC UUC CAU GAG G-3') and MMP9 siRNA (5'-AGT ACT GGC GAT TCT CTG AGG C-3') were synthesized by Genepharma (Shanghai, China) and transfected into the cells using lipofectamin 2000 (Invitogen, Carlsbad, CA).

\section{Western blot analysis}

The cultured cells or tissue samples were washed twice with phosphate buffered saline (PBS), homogenized and lysed in the RIPA buffer (Upstate, Billerica, MA, USA) for 20 min at $4{ }^{\circ} \mathrm{C}$. The lysates were centrifuged at $12,000 \mathrm{~g}$ at $4^{\circ} \mathrm{C}$ for $12 \mathrm{~min}$, and we separated the supernatants for the western blot analysis. SDS-PAGE (10\% gel) was used to separate an equal amount ( $40 \mathrm{mg}$ ) of cell lysates; then, the lysates were transferred to polyvinylidene membranes (Millipore, Billerica, MA) and probed with antibodies against the proteins of interest, including the anti-MMP9 antibody (1:1000, room temperature for 2 hours, Santa Cruz Biotechnology, Santa Cruz, CA) and $\beta$-actin antibody (1:10,000, room temperature for 1 hour, Cell Signaling Technology, Beverly, MA). Appropriate horseradish peroxidase-conjugated secondary antibodies (Santa Cruz Biotechnology, Santa Cruz, CA) were adopted to incubate the blots. An enhanced chemiluminescence (ECK) kit (Amersham ECL detection system, GE Healthcare) was used to visualize the bound antibodies, and the relative density of the target bands was determined with densitometry analysis.

\section{Wound healing assay}

A 6-well plate was used to culture the SG-63 or OSA cells. We removed the attached cells with a sterile plastic pipette tip to create a single wound in the center of the well when the cells reached $90 \%$ confluence after $24 \mathrm{~h}$. A serum-free medium was used to remove the debris. An inverted microscope was employed to visualize and photograph the cells that migrated into the wound area after $24 \mathrm{~h}$ of culturing. We performed each experiment independently at least three times.

Transwell assay

A Transwell assay was performed to estimate the effects of miR-491 on the invasion of SG-63 or OSA cells. Matrigel ( $5 \mathrm{mg} / \mathrm{ml}$ to $1 \mathrm{mg} / \mathrm{ml}$ ) was diluted into cold RPMI1640 medium without serum, and $100 \mu \mathrm{l}$ 
of the diluted matrigel was placed in the upper chamber of the Transwell with 24 wells. The Transwell was incubated for gelling for $4 \mathrm{~h}$ at $37{ }^{\circ} \mathrm{C}$, and the cells were harvested by Trypsin/EDTA and resuspended at a density of $10^{6} / \mathrm{ml}$ in media containing $1 \%$ FBS. Warm RPMI 1640 without serum was used to gently wash the gelled matrigel. Then, a 100- $\mu$ l suspension of the cells was added to the matrigel, and $600 \mu \mathrm{l}$ of RPMI 1640 containing $5-\mu \mathrm{g} / \mathrm{ml}$ fibronectin was filled in the lower chamber as an adhesive substrate. After a 24-h incubation, we removed the Transwells from the plate, and Diff-Quick solution was used for staining. A cotton swab was used to remove the non-invaded cells on the top of the Transwell, and the invaded cells were counted under a light microscope.

\section{Luciferase reporter assay}

The 3'UTR of MMP9 was PCR amplified using the following primer set: forward, 5'-GGC TCC CGT CCT GCT TTG GCA-3' and reverse, 5'-AAA GGT TAG AGA ATC CAA GTT T-3', which was subsequently sub cloned into pGL3-basic (Invitrogen, Carlsbad, CA), carrying a firefly reporter gene; the accuracy of the insert sequence was confirmed by direct Sanger sequencing. The rs105662 polymorphism (3'UTR 2182A/C) and rs9509 (3'UTR 2289 C/T) were introduced into the construct using site-directed mutagenesis (Stratagene, La Jolla, CA), respectively. A 24-well plate without antibiotics was used to plant cells at a density of $2 \times 10^{5}$ / well $24 \mathrm{~h}$ before transfection. When the confluent rate reached 90\%, Lipofecamine 2000 (Invitrogen, Carlsbad, CA) was added to the cotransfected cells with $50 \mathrm{nM}$ scramble control or miR-491 mimics and $0.6 \mu \mathrm{g}$ of firefly luciferase reporter vector containing the mutant or wild-type, in accordance with the manufacturers' protocol. To monitor the transfection efficiency, 5-ng pRL-TK plasmid, which could produce Renilla luciferase (Promega, Fitchburg, WI) was used to cotransfect each group. We washed the cells twice $48 \mathrm{~h}$ after tranfection, then passive lysis buffer (Promega, Fitchburg, WI) was used to incubate the cells; we used the Dual-Luciferase Reporter Assay System (Promega) with a GloMax 40/40 luminometer (Promega, Fitchburg, WI) to measure the firefly luciferase activity, in accordance with the manufacturers' protocol. We presented the data of three independent experiments as the mean \pm standard deviation (S.D.) and the ratio of the firefly activity of Renilla luciferase.

\section{Statistical analysis}

We employed SPSS13.0 software to perform the statistical analyses. The continuous variables were expressed as the mean \pm S.D., and we utilized an unpaired t-test or one-way ANOVA to assess the significance, as appropriate. Chi-square statistical analysis was performed to analyze the categorical variables. $\mathrm{P}<0.05$ was considered statistically significant.

\section{Results}

The location of rs1056628 and rs9509 in potential binding sites within the 3'UTR of MMP9 To elucidate the location of rs1056628 and rs9509 in the 3'UTR of MMP9, we compared the sequences of miR-491 and MMP9 3'UTR. As shown in Fig. 1, we identified a potential miR-491 binding site, and we found that the rs1056628 polymorphism $[\mathrm{A} / \mathrm{C}]$ is located in

Fig. 1. Description of $\mathrm{z}$ potential binding site of miR-491 within the 3'UTR of MMP9, as well as the location of rs1056628 and rs9509 in the 3'UTR of MMP9.

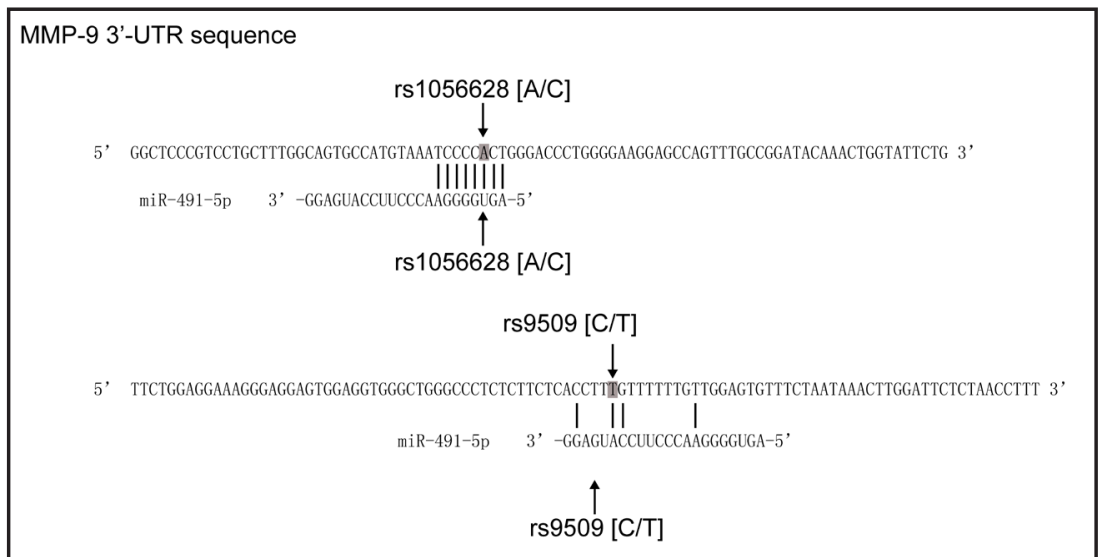




\section{Cellular Physiology Cell Physiol Biochem 2016;38:1415-1424 \begin{tabular}{ll|l} 
DOI: 10.1159/000443084 & $\begin{array}{l}\text { O 2016 The Author(s). Published by S. Karger AG, Basel } \\
\text { www.karger.com/cpb }\end{array}$ \\
\hline and Biochemistry
\end{tabular} \\ Tian/Zhang: rs1056629 Polymorphism Compromises Interaction Between MMP9 3'UTR and miR-491}

Fig. 2. A substantial decrease in luciferase activity was observed in the cells transfected with wild-type MMP9 3'UTR or MMP9 3'UTR with rs9509-C SNP compared with the scramble controls, indicating that the rs9509 polymorphism had a minimal effect on the luciferase activity. The cells transfected with MMP9 3'UTR with the rs1056628-C allele showed comparable luciferase activity compared with that of the scramble controls.
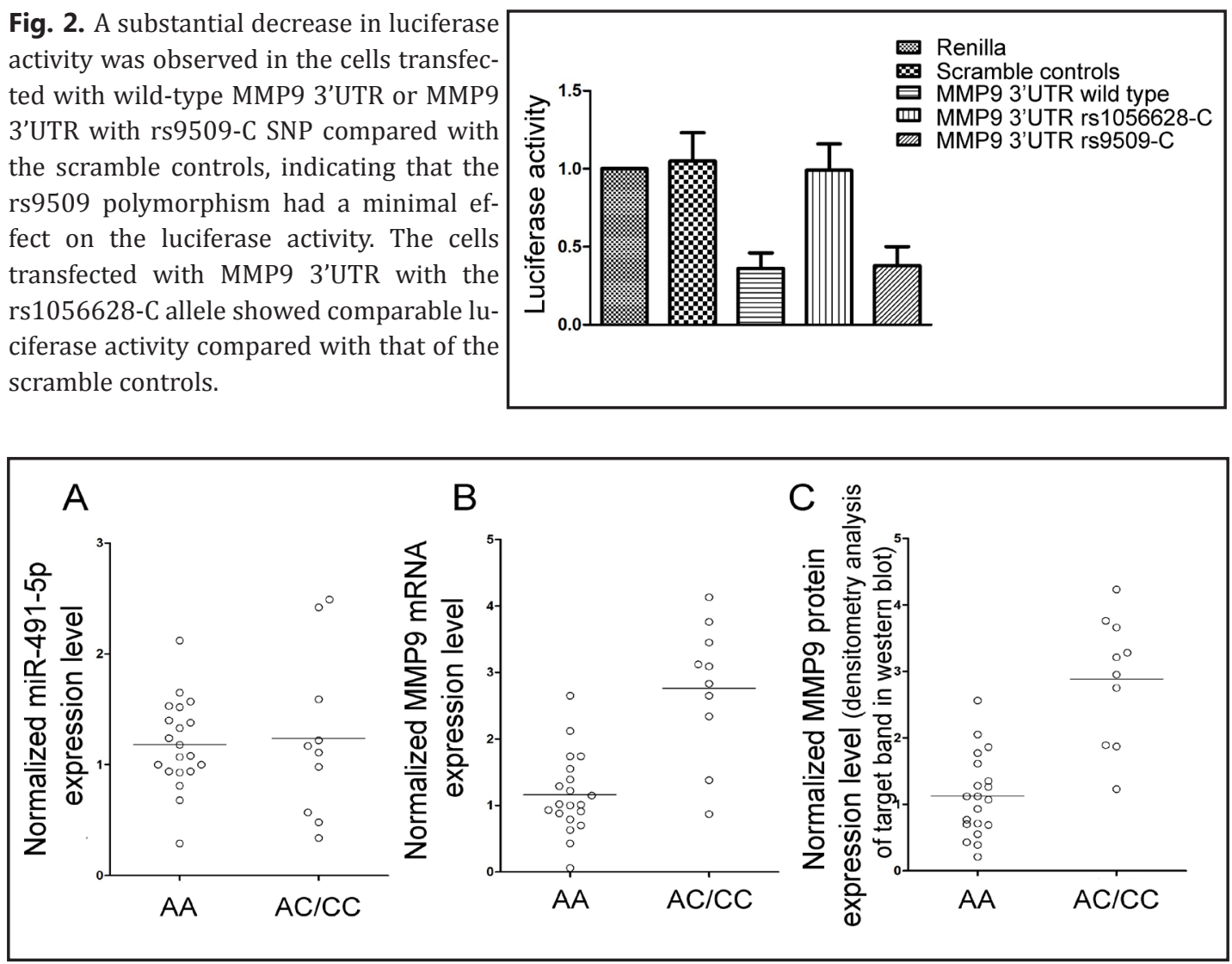

Fig. 3. The expression of miR-491 and MMP9 was determined in those samples, and we found that the expression of miR-491 was comparable between the genotype groups (A), whereas the mRNA (B) and protein (C) expression of MMP9 was much higher in the AC/CC groups than in the AA group.

the middle of the 8-nucleotide-match segment between the miR-491 and MMP9 3'UTR. In addition, another polymorphism, rs9509, was located in the 3'UTR of MMP9 107 nucleotides next to rs1056628, where there is perfect match.

Effect of rs1056628 and rs9509 on the interaction between miR-491-5p and MMP9

A dual luciferase reporter system was used to evaluate the effect of rs1056628 and rs9509 on the interaction between miR-491-5p and MMP9. Constructs carrying the wild type, rs1056628 and rs9509 were established using site-directed mutagenesis, and the constructs were transfected into the OS cells with scramble control or miR-491 mimics. As shown in Fig. 2, a substantial decrease in the luciferase activity was observed in the cells transfected with wild-type MMP9 3'UTR or MMP9 3'UTR with rs9509-C SNP when compared with the scramble controls, indicating that the rs9509 polymorphism had minimal effect upon the luciferase activity. The cells transfected with MMP9 3'UTR with the rs1056628-C allele showed comparable luciferase activity compared with the scramble controls, indicating that the minor allele of the rs1056628 polymorphism disrupted the interaction between miRNA and mRNA and abolished the effect caused by miR-491; this result suggested that the rs1056628 polymorphism was located within a binding site of miR-491 in the MMP9 3'UTR, and the presence of the minor allele of the polymorphism potentially compromised the binding between miRNA and mRNA.

Determination of miR-491 and MMP9 expression in human OS tissue samples

To further verify the effect of rs1056628 on the interaction between miR-491-5p and MMP9, we collected 30 OS tissue samples, which were genotyped for the rs1056628 


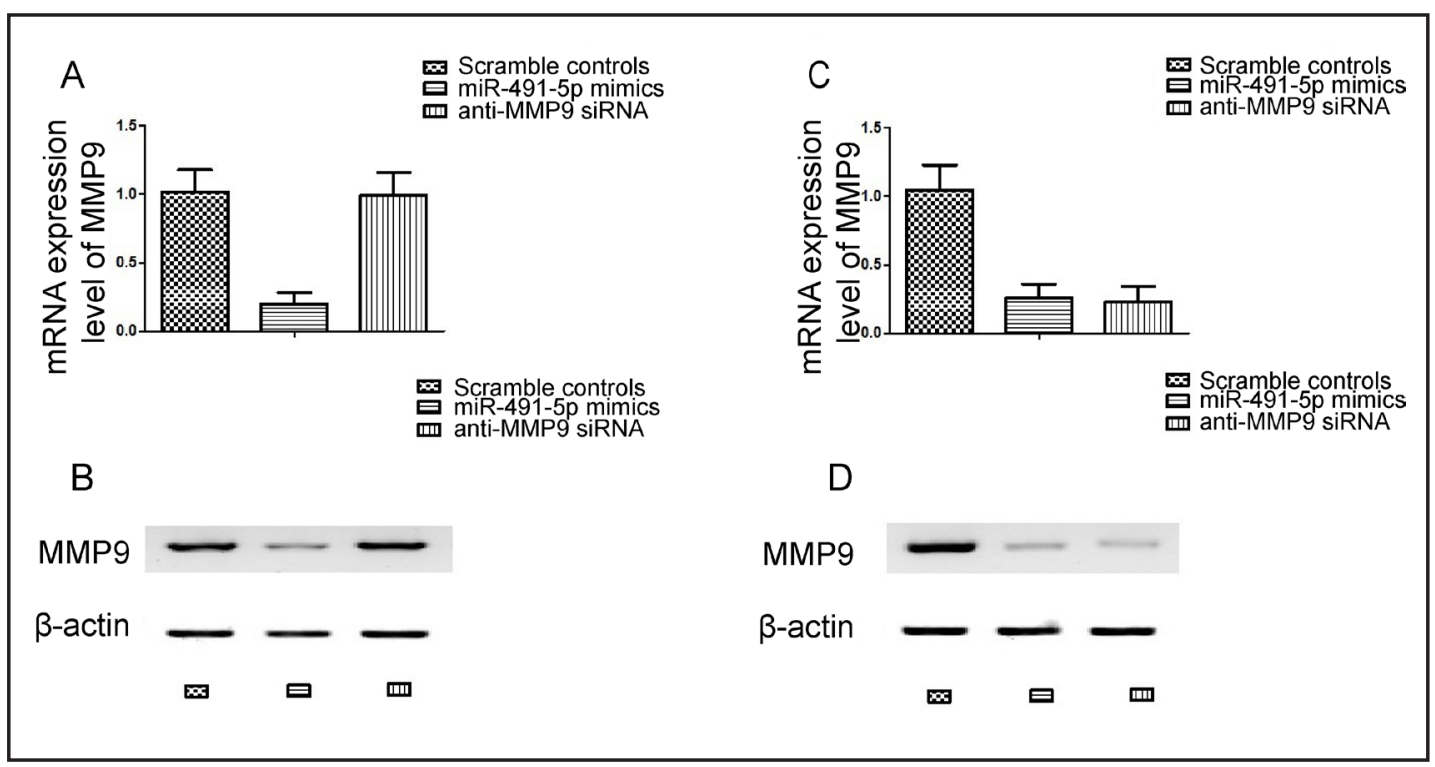

Fig. 4. In the OSA cells, only MMP9 siRNA notably reduced the expression of MMP9, whereas the introduction of miR-491 mimics left the expression of MMP9 intact compared with the results of the scramble control (mRNA of MMP9 in A and protein of MMP9 in B). Whereas in the MG-63 cells, the transfection of miR-491 mimics and MMP siRNA similarly and substantially down regulated the expression of MMP9 compared with that of the control (mRNA of MMP9 in C and protein of MMP9 in D).

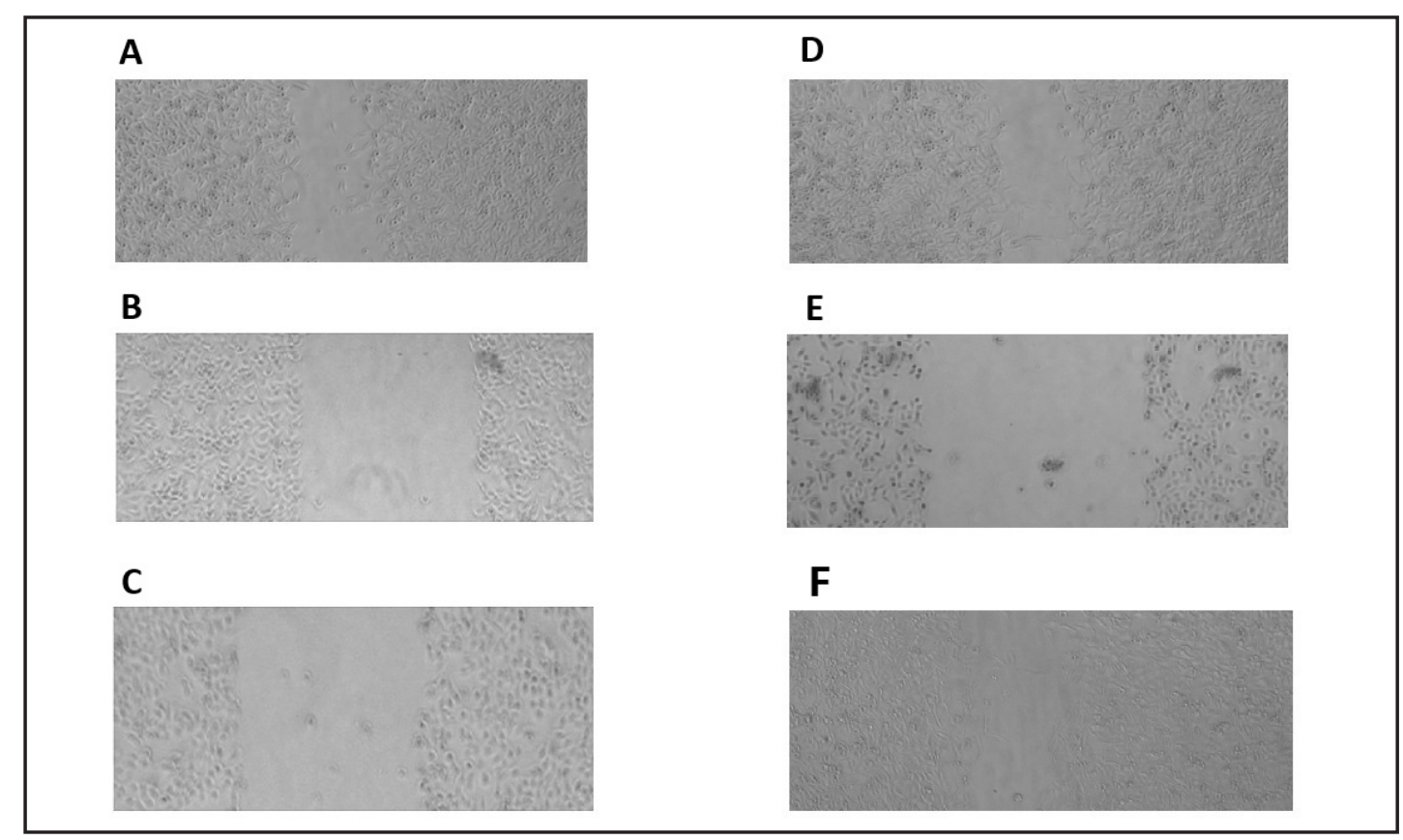

Fig. 5. The scratch test conducted in the SG-63 and OSA cells showed that transfection with miR-491 mimics (C) and anti-MMP9 siRNA (B) similarly suppressed the migratory ability of the SG-63 cells compared with that of the scramble control (A), whereas in the OSA cells treated identically, only MMP9 siRNA (E) markedly suppressed the migratory ability of the cells, and transfection with miR-491 mimics (F) had a minimal effect on it compared with the control (D).

polymorphism. The expression of MMP9 and miR-491 was determined in those samples using real time PCR and western blot analysis. As shown in Fig. 3, 30 OS tissue samples were genotyped as AA $(\mathrm{N}=20), \mathrm{AC}(\mathrm{N}=8)$ and $\mathrm{CC}(\mathrm{N}=2)$. The expression of miR-491 and MMP9 were determined in those samples, and we found that the expression of miR-491 
Fig. 6. The Transwell assay conducted in the SG-63 and OSA cells showed that transfection with miR-491 mimics and anti-MMP9 siRNA similarly suppressed the invasive ability of the SG-63 cells compared with the scramble control (A), whereas in the OSA cells treated identically, only MMP9 siRNA markedly suppressed the invasive ability of the cells, and transfection with miR-491 mimics had a minimal effect on it compared with the control (B).

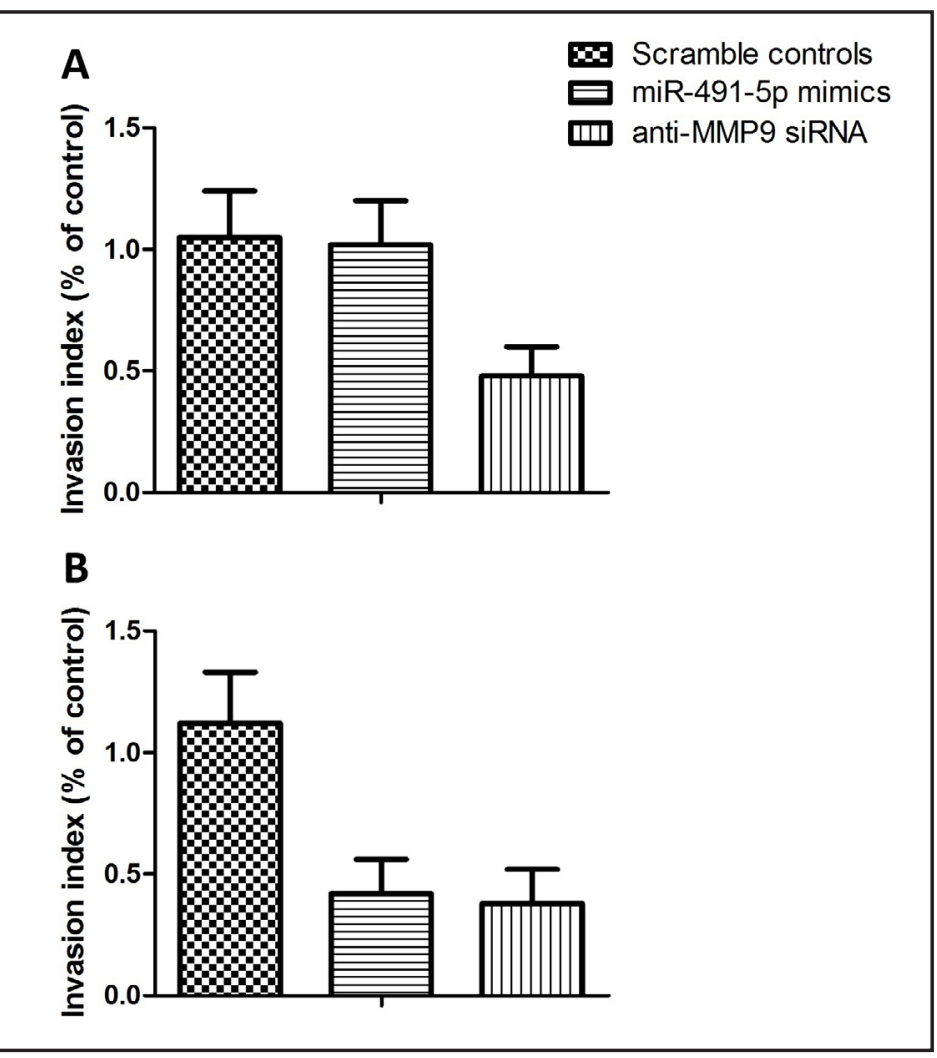

was comparable between the genotype groups; the expression of MMP9 was much higher in the AC/CC groups than the AA group, indicating that the presence of the minor allele of the rs1056628 polymorphism disrupted the binding of miR-491 with MMP9 mRNA.

The presence of the minor allele $(C)$ of the rs1056629 polymorphism abolished the inhibitory effect of miR-491, but not MMP siRNA, on the expression of MMP9

In addition, we cultured 10 OS cell lines (OSA, MG-63, Saos-2, U2OS, SARG, KPD, OHS, HAL, ZK-58, and MHM), which were further genotyped for the rs1056629 polymorphism, and selected MG-63 (genotyped as A/A) and OSA (genotyped as A/C) to be representatives for the following functional test. As shown in Fig. 4, in the MG-63 cells, the transfection of miR-491 mimics and MMP siRNA similarly and substantially down regulated the expression of MMP9; in the OSA cells, only MMP9 siRNA notably reduced the expression of MMP9, whereas the introduction of miR-491 mimics left the expression of MMP9 intact.

The presence of the minor allele $(C)$ of the rs1056629 polymorphism abolished the inhibitory effect of miR-491, but not MMP siRNA, on the expression of MMP9

Additionally, we conducted a scratch test (Fig. 5) and Transwell invasion assay (Fig. 6) to test the migratory and invasive ability of the OS cells. As shown in Fig. 5, the scratch test conducted in the SG-63 and OSA cells showed that transfection with miR-491 mimics and anti-MMP9 siRNA similarly suppressed the migratory ability of the SG-63 cells, whereas in the OSA cells that were treated identically, only MMP9 siRNA markedly suppressed the migratory ability of the cells, and miR-491 had a minimal effect on it. As shown in Fig. 6, the Transwell assay conducted in the SG-63 and OSA cells demonstrated that transfection with miR-491 mimics and anti-MMP9 siRNA similarly suppressed the invasive ability of the SG-63 cells; in the OSA cells, only MMP9 siRNA markedly suppressed the invasive ability of the cells, and miR-491 had a minimal effect on it. 


\section{Discussion}

Among the malignant bone sarcomas, OS ranks as the most common. The long-term survival rate of patients with localized disease treated with neoadjuvant therapy has improved up to $80 \%$ [16]. However, $20 \%$ of patients have detectable metastasized lesions when the disease is diagnosed $[17,18]$. The prognosis for osteosarcoma patients with metastasized lesions remains poor [17]. Determining the complexity and heterogeneity of molecular mechanism and the mechanism of OS metastasis is crucial. We identified a potential miR-491 binding site, and we found that the rs1056628 polymorphism $[\mathrm{A} / \mathrm{C}]$ is located in the middle of the 8-nucleotide-match segment between the miR-491 and MMP9 3'UTR. In addition, another polymorphism, rs9509, was found to be located in the 3'UTR of MMP9 107 nucleotides downstream (3'direction) next to rs1056628, where there is perfect match. Subsequently, we performed a dual luciferase reporter assay and found that a substantial decrease in the luciferase activity was observed in the cells transfected with wild-type MMP9 3'UTR or MMP9 3'UTR with the rs9509-C SNP when compared with the scramble controls, indicating that the rs9509 polymorphism had a minimal effect upon the luciferase activity. The cells transfected with the MMP9 3'UTR with the rs1056628-C allele showed comparable luciferase activity compared with the scramble controls, indicating that the minor allele of the rs 1056628 polymorphism disrupted the interaction between miRNA and mRNA and abolished the effect caused by miR-491.

Many human malignancies are associated with dysregulation of miRNAs, and miR-491 functions as an important tumor suppressor in many human malignancies [19, 20]. MiR491 suppresses the proliferation of colorectal cancer cells by promoting cell apoptosis via targeting Bcl-XL [21]. Additionally, miR-491 could target Bcl-XL and TP53 to induce cell apoptosis through a mitochondria-mediated pathway in pancreatic cancer [22]. Moreover, miR-491 targeted a serial of oncogenes, including GIT-1, Wnt signal and EGFR to attenuate the malignant characteristics [23-25]. Additionally, miR-491 could target G-protein-coupled receptor kinase-interacting protein 1 and MMP9 and worked as an antimetastasis gene $[19,24]$. We collected 30 OS tissue samples, which were genotyped for the rs 1056628 polymorphism. Thirty OS tissue samples were genotyped as AA $(\mathrm{N}=20), \mathrm{AC}(\mathrm{N}=8)$ and CC $(\mathrm{N}=2)$, and we found that the expression of miR-491 was comparable between the genotype groups; the expression of MMP9 was much higher in the AC/CC groups than in the AA group, indicating that the expression of miR-491 was intact, although its function was hampered by the presence of the minor allele of the rs1056628 polymorphism, which disrupted the binding of miR-491 with MMP9 mRNA.

Members of the MMP family take part in the degradation of the extracellular matrix in normal physiological processes, including tissue remodeling, reproduction and embryonic development, and they are involved in several human diseases [26, 27]. Matrix metalloproteinase-9 [MMP9] could promote invasiveness and migration of cancer cells and works as an important oncogene [28]. MMP9 degrades type IV collagen, which comprises a major part of the basement membrane, such as the basement membrane of blood vessels. Consequently, MMP9 is widely considered a good target for the development of antimetastatic drugs. The elevated expression levels of MMP9 corresponded with invasiveness and aggressiveness or a metastatic tumor phenotype [29, 30]. Foukas et al. noted that stage IIB osteosarcoma patients with elevated MMP9 expression have a significantly decreased survival rate and pulmonary metastasis, which is the primary cause of death [31]. Most studies demonstrate that an elevated MMP9 level is associated with a poor prognosis in a variety of malignancies [32, 33]. In this study, 10 OS cell lines [OSA, MG-63, Saos-2, U20S, SARG, KPD, OHS, HAL, ZK-58, and MHM) were genotyped for the rs1056629 polymorphism, and we selected MG-63 (genotyped as A/A) and OSA (genotyped as A/C) for the following scratch test and Transwell assay. In the MG-63 cells, transfection of miR-491 mimics and MMP siRNA similarly and substantially down regulated the expression of MMP9, and miRNA and siRNA clearly suppressed the migratory and invasive ability; whereas in the OSA cells, only MMP9 siRNA notably reduced the expression of MMP9, and it decreased the migratory

\section{KARGER}


and invasive ability. The introduction of miR-491 mimics left the MMP9 expression and migratory and invasive ability intact. Previous studies have demonstrated that $\gamma$-synuclein which is implicated in many types of cancer and affects metastasis is a strong upregulator of MMP9 [34]. It is a matter of further investigation to establish how polymorphism rs1056629 is involved in this mechanism of regulation. Our study has identified miR-491 as a novel metastatic suppressor in OS through the regulation of its downstream target, MMP9. Whereas the interaction between miR-491 and MMP9 could be disrupted by the presence of the minor allele of the rs1056629 polymorphism, the rs1056629 polymorphism could be a novel biomarker for predicting the occurrence of metastasis of OS.

\section{Disclosure Statement}

None.

\section{References}

1 Ottaviani G, Jaffe N: The epidemiology of osteosarcoma. Cancer Treat Res 2009;152:3-13.

2 Unni KK: Osteosarcoma; in: Unni KK, (ed): Dahlin's bone tumors (6th ed). Philadelphia, Lippincott Williams \& Wilkins, 2010, pp. 122-157.

3 Zhang B, Pan X, Cobb GP, Anderson TA: microRNAs as oncogenes and tumor suppressors. Dev Biol 2007;302:1-12.

4 Gabriely G, Wurdinger T, Kesari S, Esau CC, Burchard J, Linsley PS, Richevsky AM: MicroRNA 21 promotes glioma invasion by targeting matrix metalloproteinase regulators. Mol Cell Biol 2008;28:5369-5380.

5 Luo XJ, Tang DG, Gao TL, Zhang YL, Wang M, Quan ZX, Chen J: icroRNA-212 inhibits osteosarcoma cells proliferation and invasion by down-regulation of Sox4. Cell Physiol Biochem 2014;34:2180-2188.

6 He Y, Meng C, Shao Z, Wang H, Yang S: iR-23a functions as a tumor suppressor in osteosarcoma. Cell Physiol Biochem 2014;34:1485-1496.

7 Xu H, Liu X, Zhao J: Down-regulation of miR-3928 promoted osteosarcoma growth. Cell Physiol Biochem 2014;33:1547-1556.

8 Xu G, Wang J, Jia Y, Shen F, Han W, Kang Y: MiR-142-3p functions as a potential tumor suppressor in human osteosarcoma by targeting HMGA1. Cell Physiol Biochem 2014;33:1329-1339.

9 Yu Z, Li Z, Jolicoeur N, Zhang L, Fortin Y, Wang E, Wu M, Shen SH: Aberrant allele frequencies of the SNPs located in microRNA target sites are potentially associated with human cancers. Nucleic Acids Res 2007;35:4535-4541.

10 Moses MA: The regulation of neovascularization of matrix metalloproteinases and their inhibitors. Stem Cells 1997;15:180-189.

11 McCawley LJ, Matrisian LM: Matrix metalloproteinases: multifunctional contributors to tumor progression. Mol Med Today 2000;6:149-156.

12 Katori H, Nozawa A, Tsukuda M: Increased expression of matrix metalloproteinase-2 and 9 and human papilloma virus infection are associated with malignant transformation of sinonasal inverted papilloma. J Surg Oncol 2006;93:80-85.

13 Lakka SS, Gondi CS, Dinh DH, Olivero WC, Gujrati M, Rao VH, Sioka C, Rao JS: Specific interference of urokinase-type plasminogen activator receptor and matrix metalloproteinase- 9 gene expression induced by double-stranded RNA results in decreased invasion, tumor growth, and angiogenesis in gliomas. J Biol Chem 2005;280:21882-21892.

14 Himelstein BP, Asada N, Carlton MR, Collins MH: Matrix metalloproteinase-9 (MMP-9) expression in childhood osseous osteosarcoma. Med Pediatr Oncol 1998;31:471-474.

15 Yuan M, Zhan Q, Duan X, Song B, Zeng S, Chen X, Yang Q, Xia J: A functional polymorphism at miR-491-5p binding site in the 3'-UTR of MMP-9 gene confers increased risk for atherosclerotic cerebral infarction in a Chinese population. Atherosclerosis 2013;226:447-452. 


\section{Cellular Physiology Cell Physiol Biochem 2016;38:1415-1424 \\ \begin{tabular}{l|l|l}
\hline DOI: 10.1159/000443084 & $\begin{array}{l}\text { C) 2016 The Author(s). Published by S. Karger AG, Basel } \\
\text { www.karger.com/cpb }\end{array}$
\end{tabular}}

Tian/Zhang: rs1056629 Polymorphism Compromises Interaction Between MMP9 3'UTR and miR-491

16 Hegyi M, Semsei AF, Jakab Z, Antal I, Kiss J, Szendroi M, Csoka M, Kovacs G: Good prognosis of localized osteosarcoma in young patients treated with limb-salvage surgery and chemotherapy. Pediatr Blood Cancer 2011;57:415-422.

17 Marina N, Gebhardt M, Teot L, Gorlick R: Biology and therapeutic advances for pediatric osteosarcoma. Oncologist 2004;9:422-441.

18 Link MP, Goorin AM, Miser AW, Green AA, Pratt CB, Belasco JB, Pritchard J, Malpas J S, Baker AR, Kirkpatrick JA: The effect of adjuvant chemotherapy on relapse-free survival in patients with osteosarcoma of the extremity. N Engl J Med 1986;314:1600-1606.

19 Zhou Y, Li Y, Ye J, Jiang R, Yan H, Yang X, Liu Q Zhang J: MicroRNA-491 is involved in metastasis of hepatocellular carcinoma by inhibitions of matrix metalloproteinase and epithelial to mesenchymal transition. Liver Int 2013;33:1271-1280.

20 Wang X, Jiang F, Mu J, Ye X, Si L, Ning S, Li Z, Li Y: Arsenic trioxide attenuates the invasion potential of human liver cancer cells through the demethylation-activated microRNA-491. Toxicol Lett 2014;227:75-83.

21 Nakano H, Miyazawa T, Kinoshita K, Yamada Y, Yoshida T: Functional screening identifies a microRNA, miR491 that induces apoptosis by targeting Bcl-X(L) in colorectal cancer cells. Int J Cancer 2010;127:10721080.

22 Guo R, Wang Y, Shi WY, Liu B, Hou SQ Liu L: MicroRNA miR-491-5p targeting both TP53 and Bcl-XL induces cell apoptosis in SW1990 pancreatic cancer cells through mitochondria mediated pathway. Molecules 2012;17:4733-14747.

23 Li X, Liu Y, Granberg KJ, Wang Q, Moore LM, Ji P, Gumin J, Sulman EP, Calin GA, Haapasalo H, Nykter M, Shmulevich I, Fuller GN, Lang FF, Zhang W: Two mature products of MIR-491 coordinate to suppress key cancer hallmarks in glioblastoma. Oncogene 2015;34:1619-1628.

24 Huang WC, Chan SH, Jang TH, Chang JW, Ko YC, Yen TC, Chiang SL, Chiang WF, Shieh TY, Liao CT, Juang JL, Wang HC, Cheng A J, Lu YC, Wang LH: miRNA-491-5p and GIT1 serve as modulators and biomarkers for oral squamous cell carcinoma invasion and metastasis. Cancer Res 2014;74:751-764.

25 Hrdlickova R, Nehyba J, Bargmann W, Bose HR Jr: Multiple tumor suppressor microRNAs regulate telomerase and TCF7, an important transcriptional regulator of the Wnt pathway. PLoS One 2014;9:e86990.

26 Surgucheva I, Chidambaram K, Willoughby DA, Surguchov A: Matrix metalloproteinase 9 expression: new regulatory elements. J Ocul Biol Dis Infor 2010;3:41-52.

27 Nagase H, Visse R, Murphy G: Structure and function of matrix metalloproteinases and TIMPs. Cardiovasc Res 2006;69:562-573.

28 Egeblad M, Werb Z: New functions for the matrix metalloproteinases in cancer progression. Nat Rev Cancer 2002;2:161-174.

29 Hong S, Park KK, Magae J, Ando K, Lee TS, Kwon TK, Kwak JY, Kim CH, Chang YC: Ascochlorin inhibits matrix metalloproteinase-9 expression by suppressing activator protein-1-mediated gene expression through the ERK1/2 signaling pathway: inhibitory effects of ascochlorin on the invasion of renal carcinoma cells. J Biol Chem 2005;80:25202-25209.

30 Sato H, Seiki M: Regulatory mechanism of $92 \mathrm{kDa}$ type IV collagenase gene expression which is associated with invasiveness of tumor cells. Oncogene 1993;8:395-405.

31 Foukas AF, Deshmukh NS, Grimer RJ, Mangham DC, Mangos EG, Taylor S: Stage-IIB osteosarcomas around the knee. A study of MMP-9 in surviving tumour cells. J Bone Joint Surg Br 2002;84:706-711.

32 Liu Z, Li L, Yang Z, Luo W, Li X, Yang H, Yao K, Wu B, Fang W: Increased expression of MMP9 is correlated with poor prognosis of nasopharyngeal carcinoma. BMC Cancer 2010;10:270.

33 Folgueras AR, Pendas AM, Sanchez LM, Lopez-Otin C: Matrix metalloproteinases in cancer: from new functions to improved inhibition strategies. Int J Dev Biol 2004;48:411-424.

34 Surgucheva IG, Sivak JM, Fini ME, Palazzo RE, Surguchov AP: Effect of gamma-synuclein overexpression on matrix metalloproteinases in retinoblastoma Y79 cells. Arch Biochem Biophys 2003;410:167-176. 